\title{
Ultra large deflection of thin PZT/aluminium cantilever beam
}

\author{
Raynald Seveno and Benoit Guiffard* \\ Lunam Université, Université de Nantes, IETR UMR CNRS 6164 \\ Faculté des Sciences et des Techniques BP 92208 \\ Nantes cedex 3, France \\ *benoit.guiffard@univ-nantes.fr \\ Jean-Pierre Regoin \\ Lunam Université, Ecole Centrale de Nantes, \\ GeM UMR CNRS 6183, Nantes cedex 3, France
}

\begin{abstract}
Flexible piezoelectric cantilever beam has been realized by depositing lead zirconate titanate (PZT) thin film $(4.5 \mu \mathrm{m})$ by chemical solution deposition (CSD) onto very thin aluminium foil $(16 \mu \mathrm{m})$. The tip deflection of the beam has been measured as a function of the frequency of the applied sinusoidal voltage to the PZT film for different amplitudes. Resonance curves have been compared to a classical model of an oscillating system under sinusoidal stress with a very good agreement. Despite of weak ferroelectric properties (remnant polarization: $13 \mu \mathrm{C} / \mathrm{cm}^{2}$ ), ultra-large deflection amplitudes have been measured under very moderate applied voltage values: $750 \mu \mathrm{m} @ 10 \mathrm{~V}$ for quasi-static mode and $5 \mathrm{~mm} @ 10 \mathrm{~V}$ at the resonance frequency $(\sim 12 \mathrm{~Hz})$, which makes this PZT/ aluminium composite film very promising for highly flexible actuation applications where large displacements are wanted.
\end{abstract}

Keywords: PZT thin film; flexible film; cantilever; aluminium foil; large deflection.

Flexible piezoelectric films are gaining major interest because of the multitude of electromechanical applications that might be associated with them. Two materials are subject to extensive studies because of their best piezoelectric properties in their category: In polycrystalline form, lead zirconate titanate (PZT) thin films present transverse piezoelectric coefficients $d_{31}$ ranging from $\sim 10 \mathrm{pm} / \mathrm{V}$ to $\sim 200 \mathrm{pm} / \mathrm{V} \cdot{ }^{1-3}$ Polyvinylidene fluoride (PVDF), a semi crystalline polymer, presents the advantage of the natural flexibility of polymers, but with piezoelectric coefficients 510 times lower than the highest reported PZT thin film coefficients. ${ }^{4,5}$ While it seems difficult to improve the $d_{31}$ coefficient of PVDF already flexible, researchers have rather tried to make flexible PZT films while keeping their excellent piezoelectric properties. In general, PZT thin films are grown on stiff silicon substrates for applications in microelectronics. Some other different substrates like stainless steel, alumina, sapphire, $\mathrm{MgO}$ were also used, but they remain still too rigid for applications requiring flexible actuating micro devices. The main drawback of the synthesis of PZT thin films is the conventionally required large thermal budget $\left(600-800^{\circ} \mathrm{C}\right)$ to

*Corresponding author. obtain a complete crystallization of the perovskite phase, which makes the use of polymer substrates very limited (at least during the heat treatment). PZT films have already been deposited by sputtering onto a thermally stable polyimide film ( ${ }^{\oplus}$ Kapton), but piezoelectric properties remained low. ${ }^{6}$ Recently, piezoelectric beams have been obtained by laminating a PZT ceramic on polyethylene terephthalate (PET), yielding good vibrating properties which can be used for micropower energy harvesting applications. ${ }^{7}$ Another way that is being developed is to synthesize conventionally PZT thin film on a rigid substrate, and transfer it by various techniques (buffer, laser lift-off) on polymer like PET and polydimethylsiloxane PDMS films. ${ }^{8-10}$

In this paper, the authors present the realization of a cantilever-based PZT thin film deposited onto an ultra thin aluminium foil as a substrate and show that a very flexible actuator with low voltage-induced ultra large deflections can be obtained by this method.

The piezoelectric cantilever has been prepared by depositing a PZT thin film onto aluminium foil (with a thickness $t_{s}=16 \mu \mathrm{m}$ ) by a chemical solution deposition (CSD) process $^{11,12}$ using zirconate, titanate alkoxides and lead acetate as precursors and acetic acid as solvent. The $\mathrm{Zr} / \mathrm{Ti}$ ratio is $57 / 43$ typically used at the laboratory for other 
applications. Multiple spin-coating at $6000 \mathrm{rpm}$ leads to a PZT thickness of around $t_{p}=3 \mu \mathrm{m}$. Each layer has been crystallized at $650^{\circ} \mathrm{C}$ for $2 \mathrm{~min}$ in an open air furnace, resulting in a perovskite phase without preferred orientation. In order to apply the electric field to obtain the piezoelectric effect, aluminium top electrode of $1 \mathrm{~cm} \times 4 \mathrm{~cm}$ area ( $\sim 200 \mathrm{~nm}$ thick) has been evaporated through a shadow mask, the aluminium foil being directly used as bottom electrode. The piezoelectric film has been poled using a Sawyer-Tower circuit with a $4.7 \mu \mathrm{F}$ serial capacitor under a $200 \mathrm{kV} / \mathrm{cm}$ sinusoidal electric field at $50 \mathrm{~Hz}$. The resulting P-E measurement leads to a $13 \mu \mathrm{C} / \mathrm{cm}^{2}$ value for the remnant polarization and a $76 \mathrm{kV} / \mathrm{cm}$ value for the coercive field. The remnant polarization could be improved by increasing the applied electric field, but it would increase the risk of dielectric breakdown because of the large size of the sample and the top electrode. Moreover, the aluminium substrate is not oriented and does not promote the crystallization of the PZT film. The ferroelectric properties are also mitigated by the oxidation of the metal during the heat treatment, which forms an interfacial dielectric oxide layer at the aluminium/ PZT interface leading to a high coercive field value. For bending-mode actuation measurements, the aluminium/PZT sample has been clamped on one side resulting on an unimorph cantilever beam of $3.4 \mathrm{~cm}$ long, $1.1 \mathrm{~cm}$ wide and $20.5-\mu \mathrm{m}$ thick. A laser vibrometer (Polytech OFV 2200) has been used to measure the tip deflection at the free end of the beam.

A sinusoidal voltage with amplitude $V_{0}$ has been applied to the PZT film and the deflections at the free end of the beam as a function of the frequency of the electric field are represented in Fig. 1 for different $V_{0}$ values, from $0.7 \mathrm{~V}$ to $10 \mathrm{~V}$.

The noticeable point is the order of magnitude of the deflection at the resonance frequency, close to $12 \mathrm{~Hz}$, whatever the $V_{0}$ values. Generally, for PZT thin film-based cantilever beams, the deflection magnitude is within the nanometer or micrometer range. ${ }^{13-15}$ Due to the very flexible substrate, the size of the beam and the low ratio between the aluminium and PZT thicknesses, ultra large deflection of the beam is obtained within the millimeter range, which can be easily observed to the naked eye. The resonance behavior of the curves leads to compare the vibration of the beam to the classical model of an oscillating system under sinusoidal excitation. In this theory, the amplitude $d$ of the deflection at the end of the beam can be described by the equation ${ }^{16}$

$$
d=d_{0} / \sqrt{\left(1-\left(f / f_{0}\right)^{2}\right)^{2}+\left(f /\left(f_{0} \times Q\right)\right)^{2}},
$$

where $d_{0}$ is the quasi-static deflection value, $f$ is the frequency of the applied voltage $v=V_{0} \cos (2 \pi f t), f_{0}$ is the frequency of the oscillator of the free mode and $Q$ is the mechanical quality factor.

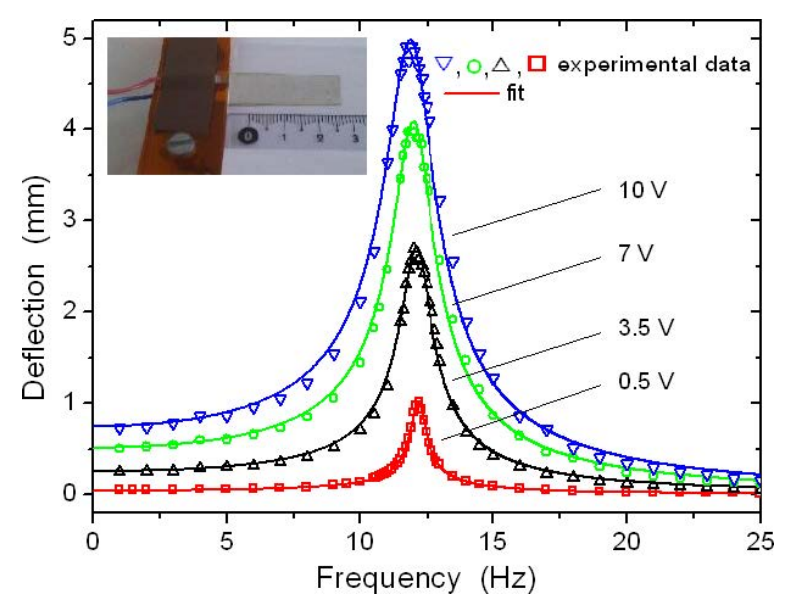

Fig. 1. Tip deflection of the cantilever beam as a function of the frequency for different amplitudes of the applied voltage and the associated fits. The inset shows a photograph of the PZT/aluminium cantilever beam and the clamp fixture.

Figure 1 also shows the very good agreement between this model and the experimental deflections. By fitting the experimental curves obtained for different applied voltages, the $Q, d_{0}$, and $f_{0}$ values have been extracted. In this model, the quality factor $Q$ should not depend on the external excitation, but it is not the case here as it is shown in Fig. 2, where the $Q$ values are represented as a function of the amplitude $V_{0}$ of the applied voltage.

The quality factor value decreases from 22 to 6.6 for $V_{0}$ starting from $0.7 \mathrm{~V}$ to $10 \mathrm{~V}$. This point has already been observed by another group ${ }^{17}$ and was attributed to the air friction. This is particularly relevant here by the use of a lightweight $(30 \mathrm{mg})$ and large $\left(3.7 \mathrm{~cm}^{2}\right)$ cantilever beam. The velocity of the vibrations increases with the amplitude of the applied voltage, then, the increasing influence of the air on the cantilever explains the decrease of the quality factor value, which reflects the mechanical frictions in the oscillator system.

In Fig. 3, are represented the maximum of the experimental deflection $d_{\max }$ at the resonance frequency, the resonance frequency $f_{r}$ as a function of the amplitude of the

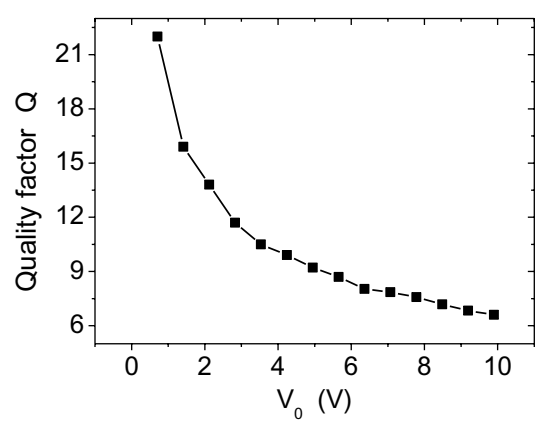

Fig. 2. Quality factor values as a function of the amplitude of the applied voltage. 


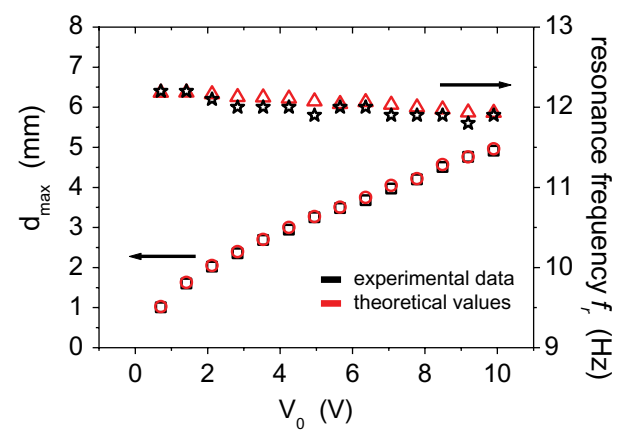

Fig. 3. Experimental and modelled values of the maximum of the deflection $d_{\max }$ and of the resonance frequency $f_{r}$ as a function of the amplitude of the applied voltage.

applied voltage and the calculated values obtained by the equations ${ }^{16}$ :

$$
d_{\max }=d_{0} \times Q / \sqrt{1-1 / 4 Q^{2}} \approx d_{0} \times Q
$$

and

$$
f_{r}=f_{0} \times \sqrt{1-1 / 2 Q^{2}} \approx f_{0} .
$$

For an applied voltage $V_{0}$ starting from $0.7 \mathrm{~V}$ to $10 \mathrm{~V}$, the measured deflection ranges from $1 \mathrm{~mm}$ to $5 \mathrm{~mm}$, which is very high compared to classical cantilevers elaborated with PZT thin films. ${ }^{18,19}$ Such large deflections can be reached because of the very thin, lightweight and flexible substrate used and larger deflection could be possibly reached with this cantilever by increasing the applied voltage, but the aluminium substrate folds under the mechanical stress. In this case, the folding is irreversible and the beam does not return to its initial position, even after removing the electric field. This is the reason why the applied voltage has been limited to $10 \mathrm{~V}$ in this study. Concerning the resonance frequency, the slight downward slope of the curve can be explained in part by the evolution of the quality factor as a function of $V_{0}$ but $f_{r}$ stays close to the $f_{0}$ value of $12 \mathrm{~Hz}$. In a first approximation, the first resonance frequency of the beam can be calculated with the following equation ${ }^{20}$ (in our study, the Young's modulus has been replaced by an effective Young's modulus taking into account the thickness of the both materials).

$$
f_{r}=\frac{\left(t_{s}+t_{p}\right) \times \alpha_{1}^{2} \times \sqrt{\left(t_{p} E_{p}+t_{s} E_{s}\right) /\left(t_{p} \rho_{p}+t_{s} \rho_{s}\right)}}{2 \pi L^{2} \sqrt{12}},
$$

where the coefficient $\alpha_{1}=1.875$, is a constant for the fundamental resonance frequency, $L$ is the length of the free part of the cantilever, $E_{p}, \rho_{p}, E_{s}$ and $\rho_{s}$ are the Young's modulus and the density of PZT and aluminium respectively. The Young's modulus values used here are: $E_{p}=95.2 \mathrm{GPa}^{19}$ and $E_{s}=69 \mathrm{GPa}$. Although the assumption on the deflection level is not validated, the deflection level should be low

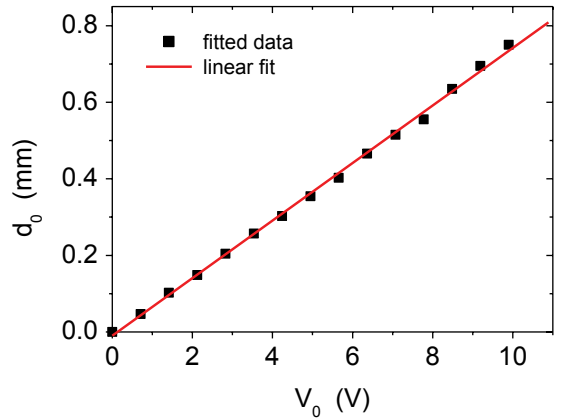

Fig. 4. Linear fit of the deflection $d_{0}$ in the quasi-static mode as a function of the amplitude of the applied voltage.

compared to the thickness of the cantilever, but here:

$$
244>\frac{d_{\max }}{\left(t_{s}+t_{p}\right)}>49
$$

the calculated resonance frequency $(12.1 \mathrm{~Hz})$ using Eq. (4) is very close to the experimental results. The piezoelectric coefficient $d_{31}$ has been calculated using the following equation $^{21}$

$d_{31}=\frac{-d_{0} \times\left(s_{s}^{2} t_{p}^{4}+4 s_{s} s_{p} t_{s} t_{p}^{3}+6 s_{s} s_{p} t_{s}^{2} t_{p}^{2}+4 s_{s} s_{p} t_{s}^{3} t_{p}+s_{p}^{2} t_{s}^{4}\right)}{3 s_{s} s_{p} t_{s}\left(t_{s}+t_{p}\right) L^{2} V_{0}}$,

where $s_{s}=1 / E_{s}$ and $s_{p}=1 / E_{p}$ and the $d_{0} / V_{0}$ ratio is obtained by the linear fit of the dependence of $d_{0}$ upon $V_{0}$ shown in Fig. 4. The calculation yields a low piezoelectric coefficient of $-10 \mathrm{pm} / \mathrm{V}$. This can be explained by the derived Sol-gel process used (for which similar $d_{31}$ value has been obtained), ${ }^{21,22}$ the nonoptimum $\mathrm{Zr} / \mathrm{Ti}$ ratio (52/48 is preferred for piezoelectric applications ${ }^{23}$ ), the low crystallization anneal $\left(650^{\circ} \mathrm{C}\right)$ yielding the weak polarization of the PZT film ${ }^{24}$ and the nonoriented metallic substrate also inducing weak ferroelectric properties. ${ }^{25,26}$ However, the deflection in the quasi-static mode $\mathrm{d}_{0}$ is quite high, ranging from $46 \mu \mathrm{m} @ 0.7 \mathrm{~V}$ to $750 \mu \mathrm{m} @ 10 \mathrm{~V}$.

In summary, this paper reports on the realization of a cantilever-based PZT thin film deposited on an ultra thin aluminium foil for bending-mode actuation. Very large deflections induced by low voltage appliance were measured, up to $5 \mathrm{~mm} @ 10 \mathrm{~V}$ at the $12 \mathrm{~Hz}$ resonance frequency. These values are much higher than those usually reported for PZT thin film-based cantilevers, which makes this PZT/aluminium composite film very promising for actuation applications where very moderate driving voltage is an asset, like in microfluidics. Besides, a good agreement was found between measured tip deflections and resonance frequency and calculated ones. To still get better electromechanical performances, future work will be devoted to the improvement of 
both the piezoelectric properties of the films by modifying the CSD process and the poling step.

\section{Acknowledgments}

The authors would like to thank Jean-Emmanuel Lechêne from Cookson SAS (Cholet, France) for supplying the shadow masks required for electrode deposition and the GeM laboratory (UMR $6183 \mathrm{CNRS}$ ) for providing the laser vibrometer.

\section{References}

1. I. Kanno et al., Appl. Phys. Lett. 70, 1378 (1997).

2. G.-T. Park et al., Appl. Phys. Lett. 80, 4606 (2002).

3. K. Sivanandan et al., Sensors Actuators A 148, 134 (2008).

4. A. G. Kepler and R. A. Anderson, Appl. Phys. Lett. 49, 4490 (1978).

5. C. Sun et al., Energy Environ. Sci. 4, 4508 (2011).

6. G. Suchaneck et al., Surface Coatings Technol. 205, S241S244 (2011)

7. A. Vásquez Quintero et al., MEMS 2012 Conference, Paris, France (2012), pp. 1289-1292.
8. J. Rho et al., IEE Electron. Dev. Lett. 31, 1017 (2010).

9. Y. H. Do et al., Sensors Actuators A 184, 124 (2012).

10. Y. H. Do et al., Sensors Actuators A 200, 51 (2013).

11. R. Seveno et al., J. Europ. Ceram. Soc. 20(12), 2025 (2000).

12. R. Seveno and D. Averty, J. Sol-Gel Sci. Technol. 68(2), 175 (2013).

13. N. Ledermann et al., Sensors Actuators A 105, 162 (2003).

14. X.-Y. He et al., J. Electroceram 21, 871 (2008).

15. T. Suzuki et al., Sensors Actuators A 125, 382 (2006).

16. F. K. Kneubühl, Oscillations and waves, Springer, ISBN 3540-62001-X, 114 (2007).

17. Q.-M. Wang et al., J. Appl. Phys. 86, 3352 (1999).

18. H. Kueppers et al., Sensors Actuators A 97-98, 680 (2002).

19. M. Dekkers et al., J. Micromech. Microeng. 23, 025008 (2013).

20. C. H. Nguyen and S. J. Pietrzko, Mech. Syst. Signal Process. 18, 929 (2004).

21. P. Luginbuhl et al., Sensors Actuators A 54, 530 (1996).

22. J. F. Shepard Jr. et al., Sensors Actuators A 71, 133 (1998).

23. S. Trolier-McKinstry and P. Muralt, J. Electroceram. 12, 7 (2004).

24. Y. Qi et al., Nano Lett. 10, 524 (2010).

25. Q. Zou et al., Appl. Phys. Lett. 77, 1038 (2000).

26. Y. W. Cho, S. K. Choi and G. V. Rao, Appl. Phys. Lett. 86, 202905 (2005). 